Ann. Sci. forest., 1978, 35 (2), 165-174.

\title{
La présence de calcaire dans le sol. Influence sur le comportement de l'Epicea commun (Picea excelsa Link.) et du Pin noir d'Autriche (Pinus Nigra nigricans Host.)
}

\author{
F. LE TACON \\ Station de Recherches sur les Sols forestiers et la Fertilisation, I.N.R.A., \\ Centre National de Recherches forestières, \\ Champenoux, 54280 Seichamps
}

Condensé de la Thèse de Doctorat ès sciences naturelles soutenue par l'auteur le 11 juin 1976 à l'Institut National Polytechnique de Lorraine, Nancy (n' ${ }^{\circ}$ CNRS A.O. 12695)

\section{Introduction}

Les affleurements calcaires occupent une grande surface en France. La présence de calcaire dans beaucoup de sols de ces affleurements pose des problèmes de mise en valeur. Seules, en effet, les espèces tolérantes au calcaire peuvent être utilisées. La présence de calcaire dans le sol provoque en effet chez de nombreuses espèces résineuses des difficultés de croissance accompagnées de troubles physiologiques.

Dans un premier temps, nous nous sommes attachés à préciser un certain nombre de propriétés des sols développés sur roche-mère calcaire dans le Nord-Est de la France. Nous nous sommes limités à l'étude de quelques facteurs parmi les plus importants : disponibilité en calcium, manganèse, azote ammoniacal et nitrique, nature de la matière organique.

Dans un deuxième temps, nous nous sommes attachés à étudier la production et la nutrition de divers peuplements d'Epicéa (espèce de type calcifuge) et de Pin noir d'Autriche (espèce tolérante au calcaire) en fonction des différentes caractéristiques des sols développés sur les plateaux calcaires du Nord-Est de la France. Ces différentes investigations nous ont amenés à émettre des hypothèses nouvelles sur les mécanismes d'action du calcaire.

Nous avons tenté de préciser expérimentalement ces mécanismes sur jeunes plants en pépinière. Nous avons ensuite été amenés à étudier le système enzymatique responsable de la réduction des nitrates et à déterminer dans quelle mesure il pouvait être affecté par la présence de calcaire dans le sol.

Enfin, des essais menés en solutions nutritives ou sur sols stérilisés nous ont permis de mettre en évidence le rôle considérable de la mycorhization dans les mécanismes de tolérance au calcaire. 


\section{I. - Influence de la présence de calcaire sur certaines propriétés essentielles du sol}

\section{1. - Nature de la matière organique}

La présence de calcaire dans un sol, dès la surface, provoque une augmentation de la teneur en matière organique totale, ainsi qu'une incorporation de matière organique sur une grande épaisseur. A ces modifications quantitatives s'ajoutent des modifications qualitatives. Nous avons pu montrer que ces modifications se traduisaient surtout au niveau de la proportion d'humine, c'est-à-dire de la matière organique non extractible par les réactifs alcalins. Il y a ainsi formation d'une forte quantité d'humine résiduelle, résistante à l'action des micro-organismes et protégée physiquement par de fines particules de carbonate de calcium. En présence de calcaire, il y aurait oxydation rapide de la lignine, ce qui ralentirait sa fragmentation, ainsi que sa minéralisation par les micro-organismes. Cette stabilisation de la matière organique humifiée entraînerait un blocage des protéines.

\section{2. - Minéralisation de l'azote}

Les modifications quantitatives et qualitatives de la matière organique, en présence de calcaire, ont des conséquences importantes sur la minéralisation de l'azote. L'ammonification nette est nettement plus faible en sol carbonaté qu'en sol décarbonaté. Le taux de minéralisation de l'azote esten effet en moyenne de 2,10 p. 100 pour les horizons $A_{1}$ des sols décarbonatés et de seulement 1,15 p. 100 pour les horizons $A_{1}$ des sols carbonatés (incubation pendant 6 semaines à $28^{\circ}$ à la capacité au champ). Cette faible ammonification en présence de calcaire semble pouvoir s'expliquer par l'augmentation de l'humine résistante à l'activité microbienne. Les pertes d'azote ammoniacal et les phénomènes de réorganisation ne sont cependant pas à exclure. Nous avons montré que l'ammonification nette dépendait étroitement de la présence ou de l'absence de calcaire, ainsi que du pH (liaison négative), et de facteurs apparentés comme le taux de saturation. Par contre, la nitrification n'apparaît liée ni à la présence de calcaire, ni au taux de saturation, ni au $\mathrm{pH}$, du moins dans la gamme des sols que nous avons étudiés ( $\mathrm{pH}$ variant de 4,5 à 8,3). Cette constance de la nitrification, contraire aux nombreuses expériences effectuées in vitro, peut s'expliquer par l'action de deux facteurs antagonistes:

- activité des organismes nitrificateurs qui augmentent lorsque le $\mathrm{pH}$ augmente ;

- diminution de la quantité d'azote transformable lorsque le $\mathrm{pH}$ croît.

$\mathrm{A} \mathrm{pH}$ élevé, c'est le deuxième phénomène qui commence à l'emporter, d'où une légère diminution de la nitrification, au-dessus de $\mathrm{pH}$ 8. La disponibilité de l'azote ammoniacal devient donc très faible en sol carbonaté, non par augmentation de la nitrification, mais par diminution de la minéralisation de l'azote en liaison avec les modifications de la nature de la matière organique. A pH élevé, comme l'avaient déjà noté de nombreux auteurs, seul l'azote nitrique est disponible, mais les mécanismes aboutissant à ce résultat sont assez différents de ceux avancés jusqu'à présent. D'autre part, la quantité d'azote minéral total mis à la disposition des plantes est beaucoup plus faible en sol carbonaté qu'en sol décarbonaté. 


\section{3. - Disponibilité en calcium des solutions du sol et $\mathrm{pH}$}

En dehors des incidences sur la minéralisation de l'azote, la matière organique joue un rôle important sur la disponibilité en calcium et le $\mathrm{pH}$ des solutions du sol. En l'absence de calcaire et de matière organique, le calcium des solutions du sol provient essentiellement des phénomènes d'échange avec le calcium du complexe absorbant. Les concentrations sont très faibles de l'ordre de 0,01 à 0,04 m.e. de calcium, pour des rapports sol eau de $1 / 5$, et même pour des sols à complexe absorbant saturé (20 m.e. de $\mathrm{Ca}^{++}$échangeable/100 g). La présence de matière organique entraîne, par l'intermédiaire des produits organiques hydrosolubles, la mise en solution de quantités importantes de calcium, pouvant atteindre $1 \mathrm{~m} . e . / \mathrm{l}$, toujours pour des rapports sol eau de 1/5. En présence de carbonate de calcium, ce calcium des solutions du sol provient, d'une part de la dissociation du carbonate et d'autre part de sa mise en solution par des produits organiques hydrosolubles plus ou moins complexes. La concentration du calcium lié aux produits organiques hydrosolubles est proportionnelle à la teneur en matière organique totale. A $20^{\circ}$ et à la pression atmosphérique, dans les horizons organiques, cette fraction de calcium liée aux produits organiques hydrosolubles est très supérieure au calcium provenant de la dissolution directe du calcaire dans l'eau. Toujours dans les mêmes conditions, c'est-à-dire à la pression atmosphérique, il y a un large recouvrement de la disponibilité en calcium des solutions du sol entre sols carbonatés et décarbonatés, en raison du rôle de ces produits organiques hydrosolubles. Ces substances organiques ont d'autre part un autre rôle important en modifiant le $\mathrm{pH}$ du sol.

Théoriquement, à la pression atmosphérique, en présence d'un excès de carbonate, le $\mathrm{pH}$ des solutions du sol est de 8,5 . Les produits organiques hydrosolubles, en raison de leur constante de dissociation plus élevée que celle des acides carboniques et bicarboniques, peuvent abaisser le $\mathrm{pH}$ jusqu'à 7 . En présence de calcaire, le $\mathrm{pH}$ et la teneur en calcium des solutions du sol, à pression de $\mathrm{CO}_{2}$ et à température constantes, dépendent donc essentiellement de la teneur en matière organique totale et de la teneur en matière organique hydrosoluble, et semblent indépendants de la teneur en calcaire total ou actif.

\section{II. - Propriétés du sol et comportement de l’Epicéa commun et du Pin noir d'Autriche}

Nous avons étudié la production et la nutrition d'un nombre important de peuplements d'Epicéa commun et de Pin noir d'Autriche, en fonction des propriétés des sols développés sur roche-mère calcaire, dans le Nord-Est de la France. Nous avons tout d'abord montré l'importance de quelques facteurs simples du sol sur la croissance de ces deux essences, et fourni ainsi aux praticiens un outil leur permettant de prévoir la production de ces deux essences, sur les différents types de sols de cette région. Nous avons ensuite tenté de préciser l'action des divers facteurs du sol, sur la croissance et la nutrition de ces deux essences, en ayant recours aux techniques modernes de traitement des données sur ordinateur. 


\section{1. - Propriétés du sol et comportement de l'Epicéa commun}

C'est ainsi que l'Epicéa apparaît comme une essence « calcifuge », ou plus exactement sensible à la présence de calcaire. En absence de calcaire dès la surface, le facteur le plus important est la profondeur de décarbonatation qui joue d'une part par l'intermédiaire des réserves en eau utile, et d'autre part par l'intermédiaire de la désaturation du complexe absorbant.

La présence de calcaire dès la surface entraîne une diminution très importante de la production, sauf en bonnes conditions d'alimentation en eau. Les variations de production en sol calcaire dépendent essentiellement des réserves en eau du sol, de la disponibilité en phosphore, et surtout du $\mathrm{pH}$. Nous avons vu que le $\mathrm{pH}$ en sol calcaire ne dépend pas de la teneur en calcaire total ou actif, mais de la teneur en matière organique, qui permet d'abaisser le $\mathrm{pH}$ par l'intermédiaire des produits organiques hydrosolubles. Or, ces substances organiques mettent en solution des quantités importantes de calcium. Ce n'est donc pas l'augmentation de la teneur en calcium des solutions du sol qui aurait un rôle néfaste sur la croissance de l'Epicéa, mais l'augmentation du $\mathrm{pH}$ du milieu, soit directement, soit indirectement par les autres facteurs qu'il conditionne. Bien qu'elle augmente la disponibilité en calcium des solutions du sol, l'augmentation de la teneur en matière organique en sol calcaire permet une amélioration de la croissance de l'Epicéa en raison de l'abaissement du pH qu'elle provoque.

\section{2. - Présence de calcaire dans le sol et nutrition minérale et azotée de l'Epicéa in situ}

L'étude de la nutrition minérale in situ apporte des précisions importantes sur les mécanismes d'action du calcaire sur le comportement de l'Epicéa. Les teneurs des aiguilles en trois éléments (manganèse, calcium et azote) présentent des différences significatives entre peuplements situés sur sols carbonatés et peuplements situés sur sols décarbonatés. La nutrition en calcium et en manganèse est très liée. La présence de calcaire entraîne dans le sol une diminution de la disponibilité en manganèse manganeux. De plus, il semble exister un antagonisme calcium manganèse, probablement au niveau de l'absorption racinaire. La teneur en manganèse des aiguilles d'Epicéa situé en sol à forte disponibilité en calcium, est donc très faible. Sur sols décarbonatés, la nutrition azotée est très supérieure à celle sur sols carbonatés. L'étude des liaisons entre nutrition minérale et croissance de l'Epicéa met en lumière le rôle primordial de la nutrition azotée. C'est l'azote qui de très loin est l'élément le plus lié à la production. Nous pouvons attribuer la faible production de l'Epicéa, en sol calcaire, essentiellement à des difficultés de nutrition azotée. La teneur en calcium des aiguilles est liée négativement à la croissance. Il semble également qu'une certaine déficience en manganèse puisse expliquer, mais très partiellement, la faible croissance de l'Epicéa en sol calcaire. La nutrition en phosphore apparaît comme très importante pour la production, mais elle n'apparaît pas liée à la présence ou à l'absence de calcaire. Enfin, le niveau de nutrition en potassium magnésium et fer ne contribue pas à expliquer les variations de croissance.

\section{3. - Propriétés du sol et comportement du Pin noir d'Autriche}

Le Pin noir en forêt a un comportement tout à fait différent de celui de l'Epicéa.

En dehors de l'influence des conditions d'alimentation en eau, peu de facteurs du 
sol apparaissent avoir une importance dans l'explication des variations de production. Le Pin noir en forêt est indifférent à la présence de calcaire dans le sol. $\mathrm{Ni}$ le $\mathrm{pH}$, ni la teneur en calcaire total ou actif, ni la disponibilité en azote minéral n'ont d'influence sur sa croissance. Un seul facteur, la teneur en phosphore assimilable du sol, a une réelle importance.

\section{4. - Présence de calcaire dans le sol ef nutrition minérale du Pin noir d'Autriche in situ}

L'étude de la nutrition minérale du Pin noir in sifu confirme ces résultats. La présence de calcaire dans le sol ne modifie que très peu la composition minérale des aiguilles. Seul, le manganèse présente une différence significative entre sols carbonatés et sols décarbonatés. On ne peut déceler aucune différence significative pour les autres éléments, et en particulier pour le calcium et l'azote. La croissance ne dépend pas de la teneur en azote des aiguilles. Le facteur le plus important apparaît encore être la nutrition en phosphore.

\section{5. - Comparaison du comportement de l'Epicéa et du Pin noir in situ}

L'Epicéa et le Pin noir ont donc un comportement tout à fait différent, in situ, visà-vis de la présence de calcaire dans le sol. Ces différences de comportement sont à mettre en parallèle avec des différences de nutrition. Chez le Pin noir, la présence de calcaire n'augmente pas la teneur des aiguilles en calcium. De plus, la teneur en calcium est toujours à un niveau bas. La teneur en calcium des aiguilles de l'Epicéa est au contraire élevée en sol acide, et augmente encore en sol calcaire. Mais c'est au niveau de la nutrition azotée que les différences sont les plus importantes.

Le Pin noir se révèle indifférent à la quantité et à la forme d'azote disponible dans les sols. Ses besoins en azote sont très faibles et semblent satisfaits avec des teneurs dans les aiguilles de l'ordre de 1 p. 100. L'Epicéa, au contraire, a des besoins importants en azote pour exprimer tout son potentiel de production, qui est nettement plus élevé que celui du Pin noir d'Autriche.

L'augmentation du pH du sol, liée à la présence de calcaire, entraîne une diminution de la minéralisation de l'azote, donc une chute de production de l'Epicéa, mais n'a aucun effet sur la production du Pin noir qui a des besoins faibles en azote. La comparaison des deux espèces laisse supposer qu'en plus de ses besoins importants en azote, l'Epicéa rencontre des difficultés d'utilisation de l'azote, et en particulier de l'azote nitrique, à $\mathrm{pH}$ élevé.

Ces difficultés de nutrition azotée semblent donc liées à des troubles du métabolisme de l'azote, lorsqu'il y a présence de calcaire dans le milieu.

\section{III. - Activité de la nitrate et de la nitrite réductase chez les deux espèces in situ}

Les premières étapes du métabolisme de l'azote lorsque l'alimentation azotée est nitrique, ce qui est le cas en sol calcaire, sont la réduction des nitrates en nitrites puis la réduction des nitrites. Nous avons donc étudié chez l'Epicéa et le Pin noir d'Autriche, 
soit en forêt sur arbres adultes, soit en pépinière sur jeunes plants, la réduction des nitrates par la nitrate réductase, et la réduction des nitrites qui semble se faire par voie non enzymatique. Nous avons pu séparer ces deux stades. En effet, la réduction des nitrates se fait aux environs de $\mathrm{pH} 7$, comme l'ont déjà montré de nombreux auteurs. La réduction des nitrites, au contraire, s'effectue à un optimum de $\mathrm{pH}$ voisin de 3,5. La comparaison de deux couples de peuplements de Pin noir d'Autriche et d'Epicéa, adultes, l'un en sol calcaire, l'autre en sol décarbonaté, met en évidence des différences entre les deux espèces. Cette différence se situe au niveau des racines, qui sont toujours mycorhizées. L'activité de la nitrate réductase augmente fortement dans les racines de Pin noir, lorsque l'on passe d'un sol acide à un sol calcaire. Au contraire, dans les racines de l'Epicéa, l'activité de la nitrate réductase diminue fortement lorsque l'on passe d'un sol acide à un sol calcaire. Ces observations permettent d'expliquer au moins partiellement les difficultés importantes de nuírition azotée rencontrées par l'Epicéa, in situ en sol calcaire. Dès la première étape du métabolisme de l'azote, c'està-dire au niveau de la réduction des nitrates en nitrites, l'Epicéa rencontre des difficultés importantes. Par contre, en forêt, le Pin noir semble réduire sans difficultés les nitrates, d'où sa tolérance en forêt aux $\mathrm{pH}$ élevés et à la présence de calcaire.

\section{IV. - Rôle de la mycorhization}

La mycorhization, comme l'a montré Krupa ef al. (1973), joue un rôle très important dans le métabolisme de l'azote chez le Pin noir d'Autriche. La nutrition azotée ayant un rôle clé dans la résistance au calcaire, nous avons cherché à déterminer quel rôle la mycorhization pouvait avoir dans ces mécanismes de résistance au calcaire.

En l'absence de mycorhization, l'Epicéa a un comportement, vis-à-vis du calcaire, sensiblement voisin de ce que l'on peut observer dans la nature. C'est ainsi qu'en solution nutritive, la présence de calcaire entraîne, chez l'Epicéa, une diminution de croissance, une augmentation de la teneur en calcium des tissus foliaires, et des difficultés de nutrition azotée. Dans ces conditions expérimentales, en l'absence de mycorhization et en milieu calcaire, nous retrouvons les mêmes difficultés d'utilisation des nitrates, que nous avions mises en évidence in situ, c'est-à-dire avec mycorhization. La mycorhization, chez l'Epicéa, ne permet donc pas d'améliorer notablement les deux particularités, qui rendent cette espèce sensible au calcaire : absorption excessive de calcium, difficultés de réduction des nitrates en présence de calcaire.

En l'absence de mycorhization, en solution nutritive ou sur sol stérilisé, le Pin noir d'Autriche, par contre, a un comportement totalement différent de son comportement dans la nature, c'est-à-dire avec mycorhization.

En présence de calcaire et sans mycorhization, il présente une chlorose s'accompagnant d'une forte accumulation de calcium dans les tissus foliaires. L'azote nitrique est mal utilisé. La protéogenèse est perturbée à deux niveaux lors du passage de l'ammonium aux acides aminés et lors de la synthèse des protéines. L'apport d'azote sous forme d'acides aminés, par voie racinaire ou foliaire, améliore sensiblement la synthèse protéique. La mycorhization, aussi bien en solution nutritive, que sur sol stérilisé, permet de rétablir une croissance normale en présence de calcaire, et corrélativement de rétablir le métabolisme de l'azote tout en permettant la régulation de la nutrition cationique. 
Influence du calcaire sur la nutrition minérale et le métabolisme de l'azote Rôle de la mycorhization chez l'épicéa ef le pin noir
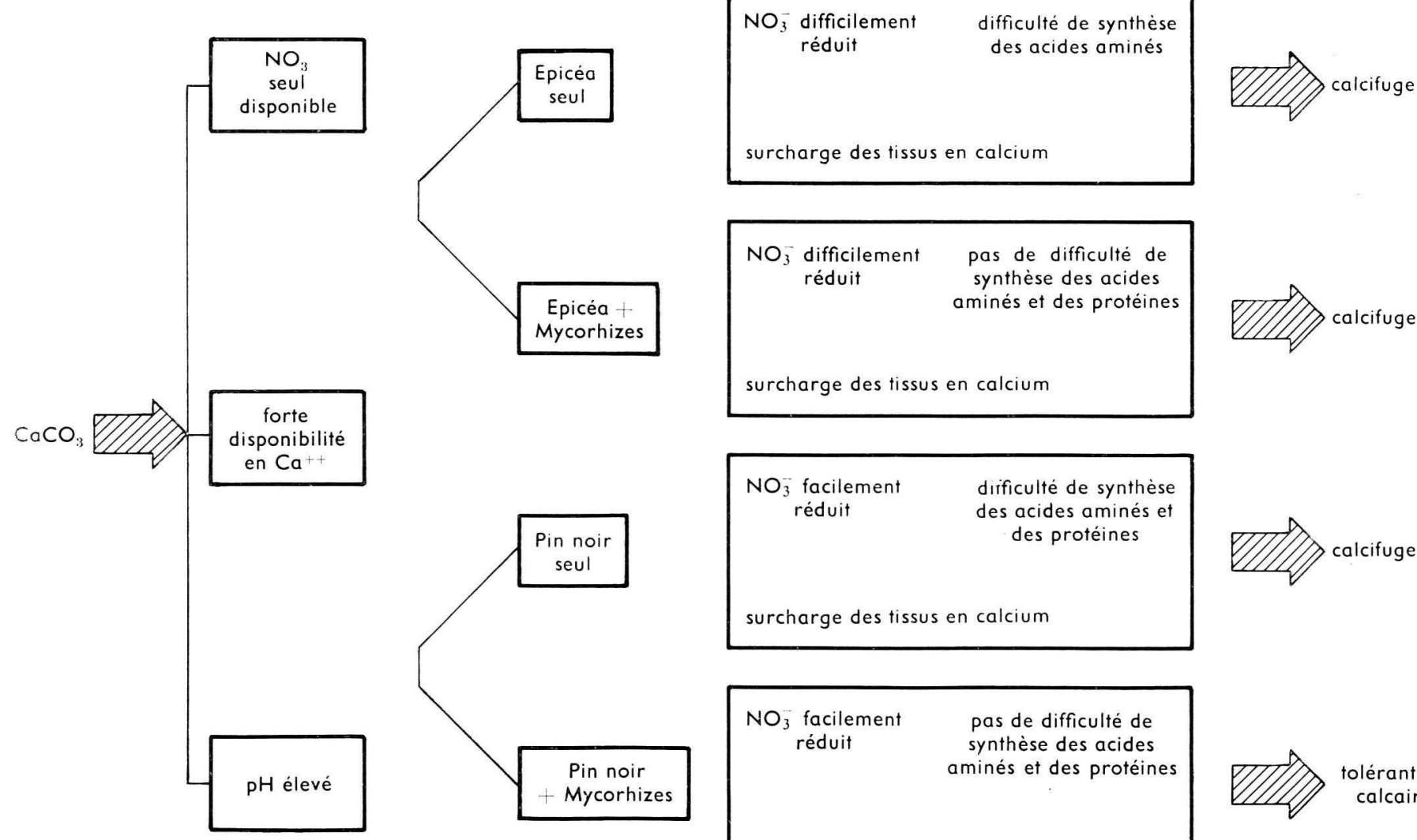

\begin{tabular}{|c|c|}
\hline $\begin{array}{l}\mathrm{NO}_{3} \text { facilement } \\
\text { réduit } \\
\text { surcharge des tis }\end{array}$ & $\begin{array}{l}\text { dificulté de synthèse } \\
\text { des acides aminés et } \\
\text { des protéines } \\
\text { en calcium }\end{array}$ \\
\hline $\begin{array}{l}\mathrm{NO}_{3} \text { facilement } \\
\text { réduit }\end{array}$ & $\begin{array}{l}\text { pas de difficulté de } \\
\text { synthèse des acides } \\
\text { aminés et des protéines }\end{array}$ \\
\hline
\end{tabular}

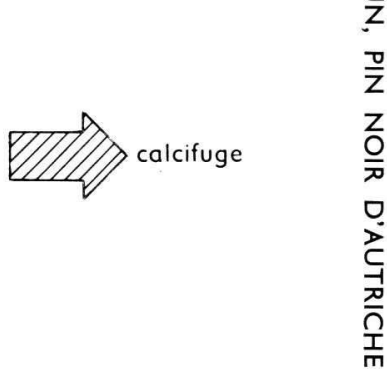

tolérant au calcaire 
Il apparaît donc que le Pin noir d'Autriche, tolérant au calcaire en conditions naturelles, est en réalité un «calcifuge» primaire. En présence de calcaire, et en l'absence de mycorhization, il présente une surcharge des tissus en cations, et des troubles graves du métabolisme de l'azote, mais contrairement à l'Epicéa, plus au niveau de la synthèse des acides aminés ou des protéines qu'au niveau de la réduction des nitrates. La mycorhization permet de rétablir un métabolisme normal de l'azote, de limiter la surcharge des tissus en cations, et par conséquent de rendre le Pin noir d'Autriche parfaitement tolérant au calcaire.

Ce caractère calcifuge des résineux, s'il se traduit en présence de calcaire par une chlorose généralisée, semblable à celle des arbres fruitiers ou de la vigne, a en réalité une cause différente. En présence de calcaire, ce sont en effet des troubles de la nutrition azotée et du métabolisme de l'azote, qui sont pour la plus grande part, et directement à l'origine des difficultés de croissance des résineux.

En conditions naturelles, il existe pour certains résineux, comme le Pin noir d'Autriche, des mécanismes régulateurs de la nutrition cationique et du métabolisme de l'azote. Ces mécanismes régulateurs sont liés à la mycorhization qui permet, par exemple au Pin noir d'Autriche, d'être secondairement tolérant au calcaire. Pour d'autres essences, comme l'Epicéa, ces mécanismes régulateurs extérieurs à la plante, sont inefficaces ou peu efficaces. Ces essences restent donc sensibles à la présence de calcaire en conditions naturelles. II n'est cependant pas impossible que des synthèses mycorhiziennes appropriées puissent permettre d'améliorer la tolérance au calcaire de nombreuses espèces résineuses réputées calcifuges.

Reçu pour publication en décembre 1977.

\section{Summary}

The presence of calcium carbonate in soil. Influence on the behaviour of Norway Spruce (Picea Excelsa Link) and Austrian Pine (Pinus Nigra nigricans Host.)

Most conifers used for reforestation in France are highly sensitive to calcium carbonate in soil. We have studied the behaviour of two species: Norway Spruce which is sensitive and Austrian Pine which is tolerant, in natural conditions.

The influence of calcium carbonate on some important properties of the soil has been analysed :

- nature of organic matter,

- availability of ammonium nitrogen and nitrate nitrogen,

- availability of calcium in soil solutions,

- availability of manganese.

\section{Nature of organic matter}

The presence of calcium carbonate from the very surface increases the level of total organic matter and the thickness of the $A_{1}$ horizon. The nature of organic matter is also modified : there is an accumulation of humine which is very resistant to microorganism activity. This humine is protected by $a$ thin film of calcium carbonate.

\section{Nitrogen mineralization}

These modifications of the nature of organic matter in the presence of calcium carbonate induce modifications in nitrogen mineralization.

Net ammonification is lower in carbonated soils than in decarbonated soils. The percentage of nitrogen mineralization is 2,10 p. 100 in $A_{1}$ horizons of decarbonated soils and 1,15 p. 100 in $A_{1}$ horizons 
of carbonated soils (incubation during 6 weeks, $18^{\circ} \mathrm{C}$, field capacity). The low ammonification of calcareous soils seems to be related to the high level of micro-organism-resistant humine.

The net nitrification is roughly the same in $A_{1}$ horizon of carbonated soils ( $\mathrm{pH} 7$ to 8,3 ) and in $A_{1}$ horizons of decarbonated soils ( $\mathrm{pH} 4,5$ to 7). There are no correlations between nitrate nitrogen production and $\mathrm{pH}$, or saturation indice or calcium carbonate content.

Thus, ammonium nitrogen availability becomes lower in carbonated soils, not because of nitrification increase, which remains constant for any $\mathrm{pH}$, but because of decrease in nitrogen mineralization (or ammonification decrease).

\section{Availability of calcium in soil solution}

Organic matter also has a great importance for availability of calcium and $\mathrm{pH}$ in soil solutions. There is a good correlation between total organic matter and water-soluble organic matter. In soil solution, a large amount of water-soluble calcium is bound with water-soluble organic matter. At $20^{\circ} \mathrm{C}$ and at atmospheric pressure, calcium bound with water-soluble organic matter is much more important in the soil solution than calcium coming from dissociation of calcium carbonate or bicarbonate.

Organic matter lowers the $\mathrm{pH}$ of these soil solutions. In case of an excess of calcium carbonate, the $\mathrm{pH}$ of a water solution is 8,5 at atmospheric pressure. In the same conditions, but with water-soluble organic matter, the $\mathrm{pH}$ of the soil solution decreases and can be as low as 7 .

\section{Soil properties and growth of Norway Spruce and Austrian Pine}

In East France, on calcareous table lands, we determined potential productivity of Norway Spruce and Austrian Pine in relation to soil characteristics. A sufficient number of sample stands were chosen so as to cover all the types of soils. In each stand, dendrometric and soils characteristics were measured as well as mineral nutrition by foliar analysis.

\section{Soil properties and growth of Norway Spruce}

Depending on different environmental conditions, the mean annual volume increament for 50 year old stands fluctuates from 3,8 to $16 \mathrm{~m}^{3} / \mathrm{ha} /$ year. The growth of Norway Spruce decreases as the content of calcium carbonate increases in soil. But its growth does not depend on calcium availability in soil solution.

\section{Presence of calcium carbonate in soils and mineral nutrition of Norway Spruce}

Nitrogen seems to be the most important element. For $\mathrm{pH}$ values below 7 , nitrogen mineralization is important and Norway Spruce can use both nitric and ammonium nitrogen.

As $\mathrm{pH}$ increases, ammonium nitrogen becomes rare and cannot be used any more and nitric nitrogen dœs not increase. The amount of total nitrogen in needles decreases and so does the yield.

On carbonated soils, the calcium content in needles is higher than on decarbonated soils.

\section{Soil properties, growth and mineral nutrition of Austrian Pine. Comparison with Spruce}

Whereas Norway Spruce presents severe growth difficulties on calcareous soils, Austrian Pine is totally indifferent to the presence of calcium carbonate in soil. It is particularly remarkable that the occurence of calcium carbonate in soil does not increase the calcium content in needles which is always low.

The production of Austrian Pine depends neither on needle nitrogen content, nor on soil nitrogen availability, or on nitrogen form $\left(\mathrm{NH}_{4}^{+}\right.$or $\left.\mathrm{NO}_{3}^{-}\right)$. Its nitrogen needs are very low and seem to be fulfilled when nitrogen content in needles is 1 p. 100 or even lower. A high nitrogen mineralization in soil does not increase its growth. Moreover, Austrian Pine in nature uses nitrate nitrogen very well on calcareous soils.

Spruce behaves differently: it has high nitrogen needs to express its growth potentiality and it seems that, besides its important nitrogen needs, it has difficulties in using nitrate nitrogen, which is the dominant form for high $\mathrm{pH}$ values. 


\section{Nitrate and nitrite reduction in roots and needles of Norway Spruce and Austrian Pine}

Nitrate reductase activity is more important in roots than in needles. Nitrite reduction seems to be non enzymatic and to be higher in foliar tissues than in root tissues.

In root tissues of Austrian Pine, nitrate reductase activity is higher on calcareous soils than on acid soils. It is the contrary for Norway Spruce. These observations can partly explain the difficulties of nitrogen nutrition of Norway Spruce on calcareous soils.

\section{Influence of mycorrhizae}

Without mycorrhizae in nutritive solutions, the behaviour of Norway Spruce is very similar to its behaviour in natural conditions : on sand, with calcium carbonate, Norway Spruce grows very slowly and uses nitrate nitrogen with difficulty. There is also an accumulation of calcium in needles as is the case in nature.

Without mycorrhizae, on sand medium with nutritive solutions, the behaviour of Austrian Pine is completely different from that it is in natural conditions : when calcium carbonate is present, Austrian Pine shows a generalized chlorosis and a very bad growth, with a high calcium accumulation in needles. Nitrate nitrogen is assimilated with difficulty. The protein synthesis is disturbed at two levels : when ammonium is transformed into amino acids, and when aminoacids are polymerised into proteins.

With mycorrhizae, in the same conditions, Austrian Pine behaves as a tolerant species and as in nature.

Thus, calcium carbonate tolerance of the Austrian Pine in natural conditions is not a genetic characteristic of the species. It is a secondary phenomenon caused by the association of the tree with some efficient fungi.

The manipulation of ectomycorrhizal fungi may be the biological tool to modifie the behaviour of forest species and to increase forest production in the future. 